\title{
Descemet Stripping Automated Endothelial Keratoplasty (DSAEK)
}

\author{
Zaman Shah ${ }^{1}$, Ibrar Hussain ${ }^{2}$, Sadia Sethi $^{3}$, Bakht Samar Khan ${ }^{4}$, Tajamul Khan $^{5}$ \\ ${ }^{1-5}$ Department of Ophthalmology, Khyber Teaching Hospital, Peshawar
}

\begin{abstract}
Purpose: The purpose of this study to analyze the visual outcome and complications of DSAEK with their management.

Study Design: Interventional case series.

Place and Duration of Study: Department of ophthalmology Khyber Teaching Hospital Peshawar, from January 2017 to April 2019.

Methods: Twenty-one patients were selected by convenient sampling method from the outpatient department of Khyber Teaching Hospital Peshawar. Informed written consent was obtained from all patients. Ethical approval of the study was obtained from institutional review board (IRB) of Khyber Medical College, in accordance with the declaration of Helsinki. All cases of DSAEK were performed by a single surgeon. We received the precut DSAEK tissue and then endoglide was used in $5(23.8 \%)$ and Busin Glide in $16(76.19 \%)$ of cases. The unfolding of the donor tissue was performed by preplaced anterior chamber maintainer using balance salt solution. Any complication either intra operative or post-operative, which happened, was recorded and managed either medically, or by appropriate surgical means.
\end{abstract}

Results: The average visual acuity before surgery was CF-1m. After DSAEK procedure, average best-corrected visual acuity was 6/36. Per-operative complications included incomplete stripping of the Descemet membrane and loss of donor button during mounting in glide. Complications in the early post-operative period were pupillary block glaucoma in 3 eyes and donor tissue dislocation in 2 eyes. Late post-operative complications included edema and non-attachment after re-bubbling, late secondary glaucoma, cystoid macular edema (CME) and interface opacification.

Conclusion: DSAEK is a promising alternative to penetrating Keratoplasty for corneal endothelial decompensation.

Key Words: DSAEK, Keratoplasty, Lamellar Keratoplasty, Endothelial Keratoplasty.

How to Cite this Article: Shah Z, Hussain I, Sethi S, Khan BS, Khan T. Descemet Stripping Automated Endothelial Keratoplasty (DSAEK). Visual Outcome, Complications and Their Management, Pak J Ophthalmol. 2020, 36 (2): 103-108.

Doi: $10.36351 /$ pjo.v36i2.977

\section{INTRODUCTION}

In Descemet stripping automated endothelial

Correspondence: Zaman Shah, Assistant Professor

Khyber Teaching Hospital, Peshawar

Email: zamanshah73@hotmail.com

Received: December 26, 2019

Accepted: March 8, 2020 keratoplasty (DSAEK), the diseased endothelium is replaced with healthy donor endothelium, Descemet membrane and part of the thin posterior corneal tissue. It is a good alternative to penetrating keratoplasty (PKP) in cases of endothelial decompensation.

In literature, DSAEK appears similar to PKP in terms of graft clarity, visual acuity, surgical risk, complications rate and endothelial cell loss but it 
seems to be superior to PKP in terms of early visual recovery, refractive stability, post-operative astigmatism wound and suture related complications and intraoperative risk. ${ }^{1}$ Some surgeons are using automated micro keratome for the preparation of donor endothelial graft, mounted on artificial anterior chamber. The procedure is known as DSAEK. At the same time many surgeons are still using manual dissection for preparation of donor tissue mounted on artificial anterior chamber and the procedure is termed as DSEK (Descemet stripping endothelial keratoplasty).

Some of the donor tissue complications are inability to separate newly prepared donor tissue from the anterior layer, excessively thickened donor posterior lenticule, donor tissue perforation and inadvertent slipping of the tissue inside of the eye. ${ }^{2}$ The most frequent complication encountered in DSAEK is donor lenticule dislocation, which can be resolved with repositioning of the graft and rebubbling. ${ }^{3}$ The proposed causes of graft detachment include patient eye rubbing and poor donor tissue dissection technique. There are reports on air induced pupillary block, primary graft failure and interface infection in early post-operative period. ${ }^{3}$ In the late post-operative period, the most important reported complications are secondary glaucoma and graft rejection. ${ }^{4}$

The purpose of this study is to evaluate the visual outcome and to analyze the per-operative and postoperative complications of DSAEK with their possible management.

\section{METHODS}

This study was performed in the Department of Ophthalmology Khyber Teaching Hospital Peshawar, from Jan 2017 to April 2019. All cases of DSAEK were performed by a single surgeon. The informed written consent was obtained from all patients. Ethical approval of the study was obtained from institutional review board (IRB) of Khyber Medical College, in accordance with the declaration of Helsinki.

The procedures were performed using the similar technique. We received the precut DSAEK tissue and then endoglide was used in $5(23.8 \%)$ and Busin Glide in $16(76.19 \%)$ cases. The unfolding of the donor tissue was performed by preplaced anterior chamber maintainer using balance salt solution.

Early post operation complications were defined as the complications that occurred within 2 months after surgery and late complications were those, which happened after 2 months of surgery. Any complication either intra operative or post-operative, were managed either medically, or by appropriate surgical means. The data was analysed using SPSS version 20 and P value of $<0.05$ was considered significant.

\section{RESULTS}

Total 21 patients were included in the study, which comprised of 5 males (23.8\%) and 16 females (76.2\%). The median age of these patients was 51.5 years (range $40-65$ ). All patients had pseudophakic corneal edema/bullous keratopathy. 20 (95.23\%) out the total had posterior chamber intraocular lens and only one $(4.7 \%)$ had anterior chamber intraocular lens.

All 21 patients had VA less than 5/60 (0.08) with most of the patients having VA of CF-1m (0.04). The average VA before surgery was CF-1m (0.03). After DSAEK procedure the best corrected VA in 8 (38.09\%) patients was 6/60 (0.1) and in $5(23.8 \%)$ patients, it was $6 / 24(0.25)$. The overall average BCVA after DSAEK was 6/36 (0.17). On paired sample t-test the $\mathrm{P}$ value was 0.001 . Table 1 shows per-operative complications. In $2(9.52 \%)$ cases, incomplete stripping of the Descemet membrane occurred. In these cases, the remaining un-stripped tissue was left as such and donor graft applied. The two most common early post-operative complications were pupillary block glaucoma in $3(14.28 \%)$ eyes and donor graft dislocation in $2(9.52 \%)$ eyes. Air induced pupillary block cases were initially treated with

Table 1: Complications of DAESK.

\begin{tabular}{|c|c|c|}
\hline Complications & $\begin{array}{l}\text { No of } \\
\text { Cases }\end{array}$ & \%age \\
\hline $\begin{array}{l}\text { Intra-operative Complications } \\
\text { Incomplete stripping of DM }\end{array}$ & 2 & 9.52 \\
\hline DSAEK detachment & 2 & 9.52 \\
\hline Loss of button in glide & 1 & 4.76 \\
\hline \multicolumn{3}{|l|}{ Early post-operative Complications } \\
\hline Donor Dislocation & 1 & 4.76 \\
\hline Air induced Pupillary glaucoma & 3 & 14.28 \\
\hline Partial donor non-attachment & 2 & 9.52 \\
\hline Blood in interface & 1 & 4.76 \\
\hline Decentration & 1 & 4.76 \\
\hline \multicolumn{3}{|l|}{ Late Post-operative Complications } \\
\hline $\begin{array}{l}\text { Edema and non attachment after re- } \\
\text { bubbling in donor dislocation }\end{array}$ & 1 & 4.76 \\
\hline Late secondary glaucoma & 1 & 4.76 \\
\hline Cystoid macular edema & 1 & 4.76 \\
\hline Interface opacification & 2 & 9.52 \\
\hline
\end{tabular}


Table 2: Comparison of "Tan endoglide" Vs "Busin Glide".

\begin{tabular}{lccllcc}
\hline & No. & \%age & Delivery of Donor Tissue & Site of Incision & Iris Prolapsed & Incision Size \\
\hline Tan endoglide & 5 & $23.8 \%$ & Difficult & Scleral & None & $4.5 \mathrm{~mm}$ \\
Busin Glide & 16 & $76.19 \%$ & Comparatively easy & Corneal & None & $4 \mathrm{~mm}$ \\
\hline
\end{tabular}

intravenous injection of mannitol and oral acetozolamide $250 \mathrm{mg}$ - four times a day and pupillary dilatation. Air bubble was not removed in any of the cases. In our case series, one donor graft dislocation happened in a case with AC IOL. This case was managed by pupillary dilatation, repositioning and rebubbling on first post-operative day. The second case was treated by repositioning and re-bubbling. Decentration occurred in one $(4.76 \%)$ case, which was also managed with repositioning and re-bubbling.

The most common late post-operative complication was corneal edema and non attachment of DSAEK tissue in one $(4.76 \%)$ case. The other important complication was late secondary glaucoma in one $(4.76 \%)$ case, cystoid macular edema (CME) occurred in one $(4.76 \%)$ eye and interface opacification in $2(9.52 \%)$ eyes. CME was treated with sub-tenon injection of triamcinolone acetonide and Nepafenec eye drop 3 times a day for 3 months. There was an improvement in vision with resolution of macular edema. In this study the rate of complications was more in cases where venting incision was done. Edema and non-attachment after re-bubbling was seen in one $(4.76 \%)$ case of venting incision. While these complications were not seen in non-venting cases. Post-operative scarring at the venting site and epithelial ingrowths were not seen in any case.

Table 2 shows comparison of Busin glide and Tan endoglide in the DSAEK procedure. We did 5 (23.8\%) cases with Tan endoglide which was found difficult for delivery and time consuming and 16 (76.19\%) cases were performed with Busin glide which was comparatively easy and less time consuming. The site for incision was selected as $4.5 \mathrm{~mm}$ scleral with Tan endoglide and $4.00 \mathrm{~mm}$ corneal for Busin glide. No iris prolapse occurred with any of the two glides.

The overall medium endothelial cell loss (ECL) after 6 months was $16.7 \%$. It was $20.3 \%, 32.2 \%$ after 12 months and 18 months follow-up (Figure 1). However, the ECL has not been analyzed independently with different groups of patients and with or without complications.

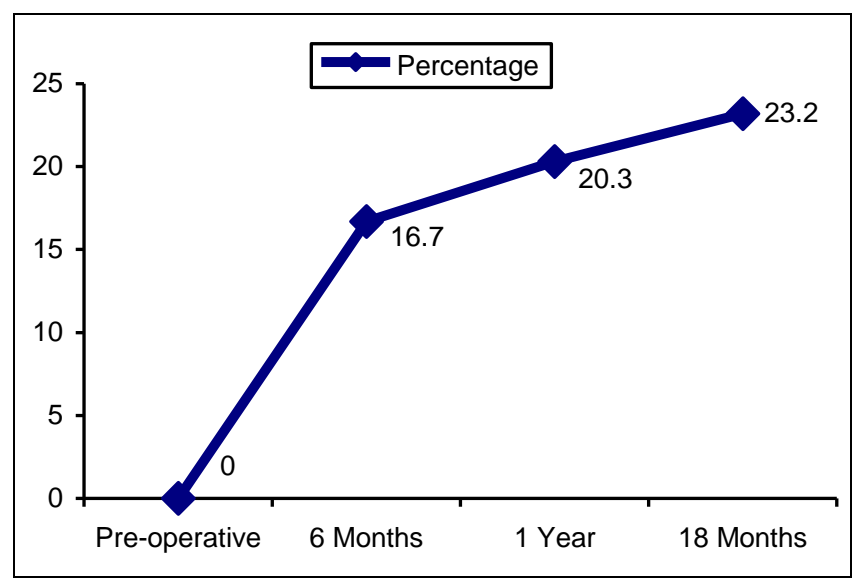

Fig. 1: Endothelial Cell Loss in \% Age with Time.

\section{DISCUSSION}

The DSAEK offers an effective and efficient alternative to traditional PKP for the treatment of corneal endothelial dysfunctions. The different complications of DSAEK are pupillary block by air, donor dislocation, graft failure, secondary glaucoma and graft rejection. The potential causes of donor dislocation include; presence of interface viscous fluid or air, patient squeezing and eye rubbing. There are complications with preparation, handling and insertion of donor lamellar tissue into the anterior chamber of the recipient. Most of the reported complications are with automated dissection of the donor tissue but evidence is lacking about management of these complications.

As previously described, pupillary block by air is an important complication of DSAEK procedure. The reported incidence of pupillary block varies between $0.5 \%$ and $13 \%$ in different series. ${ }^{5}$ This is due to the displacement of an excessively large air bubble. In our series, the overall frequency was $4.76 \%$. This complication can be prevented by placing a freely mobile air bubble and putting a drop of cycloplegic at the end of surgery.

Donor dislocation is one of the most important complications and the rate varies from $0 \%$ to $82 \%$, with an average dislocation rate of $14.5 \%$. The graft dislocation may represent either fluid in the interface 
of an otherwise well positioned graft or complete dislocation into the anterior chamber. It is interesting to notice that the incidence of this unique complication is reduced with experience and the same author had reported $8 \%$ dislocation rate in $2008 .{ }^{6}$ Price reported a dislocation rate of $50 \%$ on the first 10 eyes undergoing DSAEK, which was reduced to $13 \%$ in the next 126 cases after changing the procedure to include face up position after surgery and smoothening of the corneal surface. ${ }^{7}$ With experience and time, the dislocation rate is reduced. The results of dislocation management are also satisfactory with a success rate of $72.3 \%$ that is comparable with other published series. ${ }^{8}$

The published studies showed rate of primary graft failure (PGF) from $0 \%$ to $29 \%$, with an average PGF rate of $5 \% .^{9}$ PGF has been linked with poor surgical technique of DSAEK and related excessive iatrogenic intraoperative manipulation of donor endothelial cells. ${ }^{10}$ In our series, no case of PGF was recorded, probably due to less manipulation of DSAEK button.

Published reports on secondary glaucoma after DSAEK are between $0 \%$ and $15 \%$, with an average of $3 \%$. In our series, the incident of secondary glaucoma was $4.76 \%$ and the commonest cause of this late secondary glaucoma was topical corticosteroid.

Among reviewed studies the endothelial rejections rates varied from $0 \%$ to $45 \%$, with an average rejections rate of $10 \%$ with the follow-up ranging from 3 to 24 months. ${ }^{10}$ In our series the rejection rate was $0 \%$.

Epithelial ingrowths, interface opacification and interface hemorrhage are less common complications in our series and these are comparable with reported studies. ${ }^{11,12}$ Among theses, interface opacity is one of the important reasons for repeat endothelial keratoplasty reported by Letko et al, following 1050 consecutive DSAEK cases in 5 years. ${ }^{13}$ Interface fibrosis was also described histopathologically in failed DSAEK cases where PKP procedure was performed later on.

The incomplete removal of DM as a cause of partial graft detachment in DSAEK has been reported. ${ }^{14}$ In our series, partial donor detachment happened in two cases and with time they attached completely, which was because of incomplete stripping of DM in two cases. In both cases the graft was initially attached in more than two third areas.

Postoperative cystoid macular edema developed in one $(4.76 \%)$ eye, which resolved with topical nonsteroidal anti-inflammatory agent and sub-tenon triamcinolone acetonide injection. This is again comparable with the previous reports. ${ }^{15}$

Late secondary donor failure due to chronic endothelial cell loss is a question in DSAEK procedure. The reported late graft failure varies between 0 and $45 \%$ after 01 year with an average of $6 \%$ in first year. ${ }^{16}$ In our series the study duration is up to 18 months and the endothelial cell loss was $23.2 \%$. Late graft failure was more in pseudophakic eye with AC IOLs than with PC IOL (11.7\% versus $2.4 \%$ ). Previous studies have also shown that endothelial cell loss (ECL) in DSAEK in Pseudophakic eyes with AC IOLs is higher and the graft failure was $16 \%$ with up to 30 months follow up. ${ }^{15}$ Therefore, DSAEK surgery in patients with AC IOL remains controversial. As the published report of DSAEK beyond 5 years are few in number, so long term graft clarity with DSAEK is yet to be determined. Retanasi et al showed that only four (7.8\%) eyes had a late donor failure among $5 \%$ cases in their longest follow up of more than 5 years. ${ }^{16}$ The author states that long term results of DSAEK were excellent. The grafts were clear despite lower than normal endothelial cell count. The total graft failure in this large series was $31(7.2 \%)$ eyes. The failed DSAEK cases, early or late can be managed by redo procedure in majority $(54.8 \%)$ of cases.

The infection following DSAEK procedure, either in the form of interface kerititis and endophthalmitis in early post operative period or delayed kerititis after 03 months is always serious. ${ }^{17-19}$ In our study, at the end of 18 months follow up, not a single case of infection was seen. As a fairly new procedure the relative experience of surgeons in earlier cases may account for more graft manipulation and ECL during surgery. In addition, the DSAEK in certain indications have more complications then clear case of PC IOL related Pseudophakic Bullous Keratopathy or Fuchs endothelial dystrophy. The different conditions are aphakic AC IOL related pseudophakic bullous keratopathy (PBK); post penetrating keratoplasty (PKP) failed graft, congenital hereditary endothelial dystrophies (CHED) and irido-corneal endothelial syndrome (ICES). In aphakic cases there are reports of posterior dislocation of the donor disc into the vitreous cavity with or without retinal detachment. ${ }^{20}$ Other difficult cases include vitreous in anterior chamber, previous large peripheral iridectomy, large YAG laser capsulotomy even in the presence of PC IOL and a 
large filtration bleb. Extra care and precautions are required in these difficult cases.

\section{CONCLUSION}

In conclusion, the DSAEK is an exciting and promising alternative to the traditional PKP. Like other corneal transplantation surgeries, the learning curve is steep and the potential for complication is significant during first few cases. Both operative and post-operative complications do occur in DSAEK and increase with the long postoperative follow up, but all are within an acceptable limit. The re-DSAEK can be easily performed in most of the failed cases with satisfactory results.

\section{Ethical Approval}

The study was approved by the Institutional review board/Ethical review board.

\section{Conflict of Interest}

Authors declared no conflict of interest

\section{REFERENCES}

1. Lee WB, Jacobs DS, Msch Kanfinan SC. Descemet's stripping endothelial keratoplasty: safety and outcomes. A report by American Academy of Ophthalmology, 2009; 116: 1818-1830.

2. Glasser DB. Tissue complication during endothelial keratoplasty. Cornea. 2010; 29: 1428-1429.

3. Terry MA, Shamie N, Chen ES. Endothelial keratoplasty for fuchis dystrophy with cataract: complication and clinical results with the new tripling procedure. Ophthalmology, 2009; 116: 631-639.

4. Terry MA, Hoar KL, Wall J. Histology of dislocations in endothelial keratoplasty (DSAEK and DALK): a laboratory based surgical solution to dislocation in 100 consecutive DSEK cases. Cornea, 2006; 25: 926-932.

5. Koenig SB, Covert DJ. Early results of small-incision Descemet's stripping and automated endothelial keratoplasty. Ophthalmology, 2007; 114: 221-226.

6. Basak SK. Descemet stripping and endothelial keratoplasty in endothelial dysfunctions: Three-month results in 75 eyes. Indian J Ophthalmol. 2008; 56: 291296.

7. Price MO, Gorovoy M, Benetz BA. Descemet's Stripping automated endothelial keratoplasty outcomes compared with penetrating keratoplasty from the ornea Donor Study. Ophthalmology, 2010; 117: 438-444.
8. Chaurasia S, Vaddavalli PK, Ramappa M. Clinical profile of graft detachment and outcomes of rebubbling after Descemet stripping endothelial keratoplasty. Br J Ophthalmol. 2011; 95: 1509-1512.

9. Shih CY, Ritterband DC, Rubino S. Visually significant and non-significant complications arising from Descemet stripping automated endothelial keratoplasty. Am J Ophthalmol. 2009; 148: 837-843.

10. Ashar JN, Madhavi Latha K, Vaddavalli PK. Descemet's stripping endothelial keratoplasty (DSEK) for children with congenital hereditary endothelial dystrophy: Surgical challenges and 1-year outcomes. Graefes Arch Clin Exp Ophthalmol. 2012; 250: 13411345.

11. Ku BI, Hsieh YT, Hu FR, Wan IJ, Chen WL, Hou YC. Endothelial cell loss in penetrating keratoplasty, endothelial keratoplasty, and deep anterior lamellar keratoplasty. Taiwan J Ophthalmol. 2017; 7 (4): 199204. Doi:10.4103/tjo.tjo_55_17

12. Schmitt AJ, Feilmeier MR, Piccoli FV. Interface blood after Descemet stripping automated endothelial keratoplasty. Cornea, 2011; 30: 815-817.

13. Letko E, Price DA, Lindoso EM. Secondary graft failure and repeat endothelial keratoplasty after Descemet's stripping automated endothelial keratoplasty. Ophthalmology, 2011; 118: 310-314.

14. Lass JH, Gal RL, Dontchev M. Cornea Donor Study Investigator Group. Donor age and corneal endothelial cell loss 5 years after successful corneal transplantation. Specular microscopy ancillary study results. Ophthalmology, 2008; 115: 627-632.

15. Gupta PK, Bordelon A, Vroman DT. Early outcomes of Descemet stripping automated endothelial keratoplasty in pseudophakic eyes with anterior chamber intraocular lenses. Am J Ophthalmol. 2011; 151: 24-28.

16. Ratanasit A, Gorovoy MS. Long-term results of Descemet stripping automated endothelial keratoplasty. Cornea, 2011; 30: 1414-1418.

17. Sengupta J, Khetan A, Saha S. Bacterial keratitis after manual Descemet stripping endothelial keratoplasty-a different pathophysiology? Eye Contact Lens, 2010; 36: 62-65.

18. Lee WB, Foster JB, Kozarsky AM. Interface fungal keratitis after endothelial keratoplasty: A clinicpathological report. Ophthalmic Surg Lasers Imaging, 2011 Apr 14: 42. Online e44-48. Doi: 10.3928/15428877-20110407-01.

19. Ortiz-Gomariz A, Higueras-Esteban A, GutiérrezOrtega ÁR. Late-onset candida keratitis after Descemet stripping automated endothelial keratoplasty: Clinical and confocal microscopic report. Eur J Ophthalmol. 2011; 21: 498-502. 
20. Afshari NA, Gorovoy MS, Yoo SH. Dislocation of the donor graft to the posterior segment in Descemet stripping automated endothelial keratoplasty. Am J Ophthalmol. 2012; 153: 638-642.

\section{Author's Designation and Contribution}

Dr. Zaman Shah; Assistant Professor: Research design, Data collection, Manuscript writing, Final review.
Dr. Ibrar Hussain; Head of Department of Ophthalmology: Research design, Final review.

Dr. Sadia Sethi; Professor: Research design, Final review.

Dr. Bakht Samar Khan; Associate Professor: Research design, Final review.

Dr. Tajamul Khan; Associate Professor: Research design, Final review.

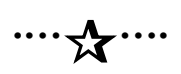

